\title{
Design and Implementation of Strain Strengthening Automation Control System for Austenitic Stainless Steel Pressure Vessels
}

\author{
Lanqing Tang ${ }^{1, a}$, Huifang Li ${ }^{1, b}$, Qi Zhang ${ }^{2, c}$, Ping Qiu ${ }^{3, d}$ and Caifu Qian ${ }^{1, f^{*}}$ \\ ${ }^{1}$ Beijing University of Chemical Technology, Beijing 100029, China \\ ${ }^{2}$ Zhangjiagang Institute of Measurement and Testing Technology 215600, China \\ ${ }^{3}$ Hebei SQD Cryogenic Equipment Co, Ltd, Hebei 065700, China \\ alanqingtang@hotmail.com, ${ }^{\mathrm{b}}$ lihf@mail.buct.edu.cn, 'Zhangqi8583@163.com, \\ dPqiu@mail.sqdcryo.com , 'qiancf@mail.buct.edu.cn
}

Keywords: austenitic stainless steel pressure vessels; strain strengthening; automation system Abstract. According to the technology requirements of strain strengthening, hardware instruments, such as upper computer, PLC , A/D converter, D/A converter, frequency converter, sensors and software components such as configure software, PLC programming software were adopted to design and develop a strain strengthening automation system for austenitic stainless steel pressure vessels. The automation system can realize a wide range of functions such as control program loading, data acquisition, strain strengthening process monitoring, experiment data saving and printing, pressurization rates controlling as well as measurements of radial strain and residual volumetric deformation. The actual practice shows the system has characteristic of running stabilization, high control accuracy, high-degree security and having the ability of automation control for strain strengthening process.

\section{Introduction}

With the development of technology and growing energy demand, cryogenic vessels have been an increasingly important way for gas storage [1]. Austenitic stainless steel, which is widely used in cryogenic vessel production, can be enhanced through strain strengthening process [2]. However, most factories in China prefer to choose manual operation, which can be regarded as a time-consuming and labor-wasting process [3].

According to the technology requirements of strain strengthening, hardware instruments, such as upper computer, PLC , A/D converter, D/A converter, frequency converter, sensors and software components such as configure software, PLC programming software were adopted to design and develop a strain strengthening automation system for austenitic stainless steel pressure vessels. In general, the automatic control system which can replace traditional manual process has several benefits such as ensuring product quality, realizing the scientific management in strain strengthening process.

\section{Hardware design of automatic control system}

The system hardware is composed of IPC, PLC, Sensors, A/D converter module, D/A converter module, pressure pumps, valves, frequency converter and other site instruments and gauges.Fig.1 demonstrates the hardware structure of automatic control system. SIMATIC IPC installed with configuration software Kingview 6.01 is adopted as the upper computer of the whole system. Meanwhile, FX2N-32MR-001 PLC, which can control scene components during strain strengthening process, is used to serve as a down computer of the system [4]. A/D converter module can convert the fault data and store them in a PLC while D/A converter module can convert digital value into analog value to control the frequency converter. Weight sensors, displacement sensors and pressure sensors are adopted to gather on-the-spot signals. Besides, pressure valves and pumps are controlled by switch signals from PLC. 


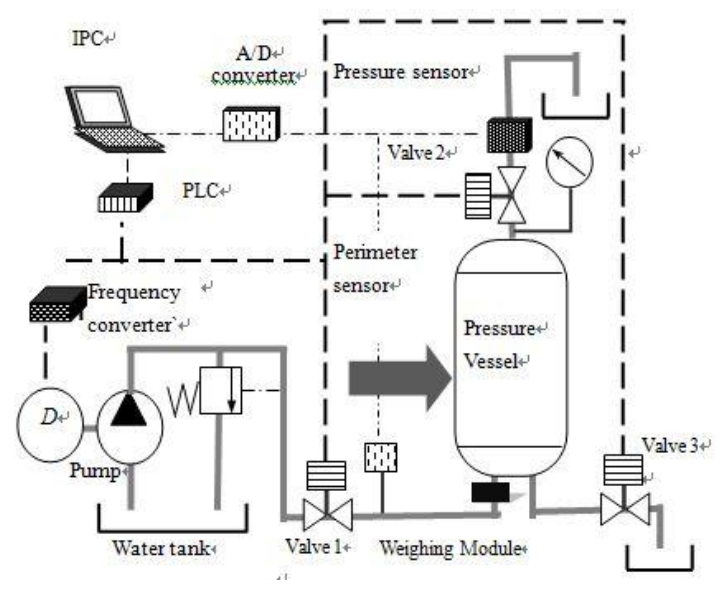

Fig.1 structure of automatic control system

\section{Software design of the automatic control system}

\section{Monitor interface design}

Configuration software Kingview 6.01 is used to create and design motoring interface for strain strengthening monitoring system. In general, Kingview software can realize many functions such as data acquisition, on-the-spot control, process animation, report saving and printing, data transmission, real-time curves and history curve [5].

In this article, monitor interface including main monitoring screen, parameter setting interface, real-time trend curve and history trend curve and report interface is established to meet the requirements of monitoring demands.

\section{Parameter setting interface}

Parameter setting interface is created by configuration software to make it easy for user to set strain-strengthening parameters. Related key parameters can be configured through parameter setting interface and then be written into data registers in FX2N PLC.Fig.2 demonstrates the parameter setting interface of automatic system.

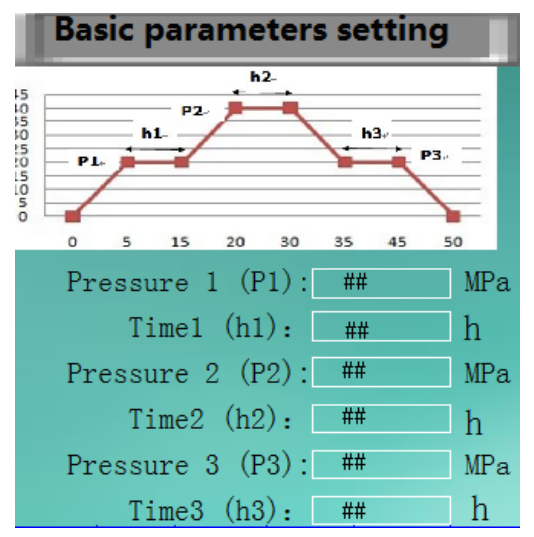

Fig.2 parameter setting interface

\section{The main monitoring screen}

The main monitoring screen can provide real-time monitoring of the whole process of strain strengthening. As shown in Fig.3, it realizes many functions such as the display of significant parameters, the acquisition from sensor data, long-distance control of executive components and the switch between different interfaces. 


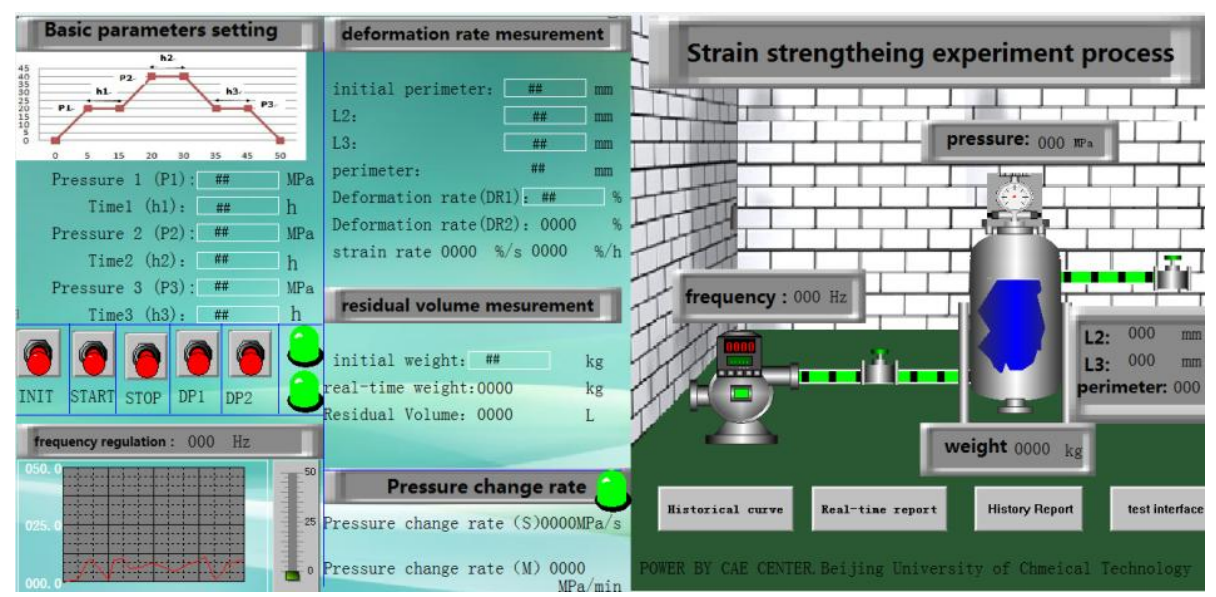

Fig.3 monitor main screen

\section{Real-time trend curve and history trend curve}

Real-time trend curve and history trend curve can be switched from the main monitoring interface by clicking the toggle buttons. Real-time trend curve can demonstrate the real-time trends of parameters such as weight, perimeter, pressure, deformation rate and frequency. Likewise, user can view history trend of some specific parameters. Fig.4 presents the pressure-time trend cure during the strain strengthening process.

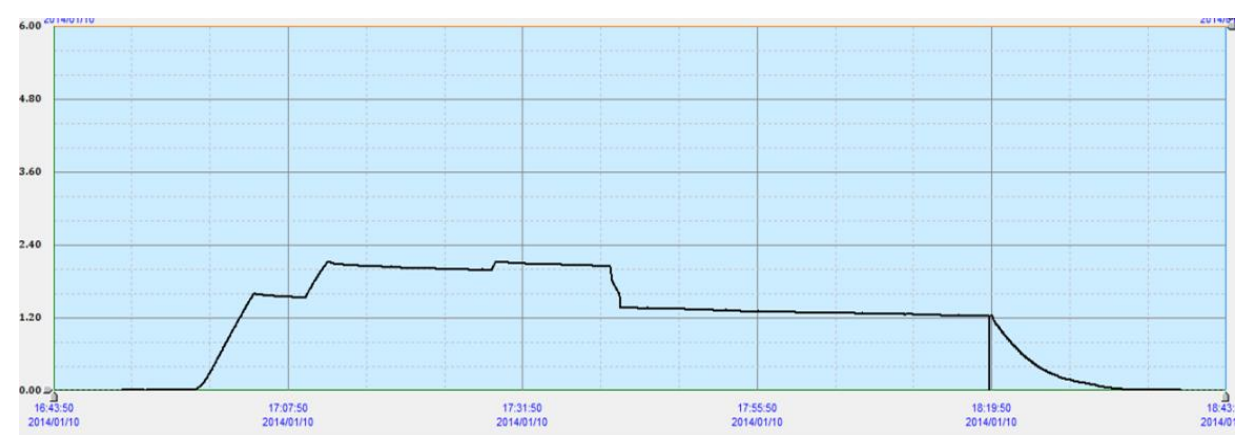

Fig.4 pressure- time history trend curve

\section{Report interface}

Real-time report and history report can be created in the report interface. In addition, users could print or save report by clicking the corresponding buttons.Fig.5 presents real-time report during strain strengthening process.-time trend curve and history trend curve can be switched from the main monitoring interface by clicking the toggle buttons.

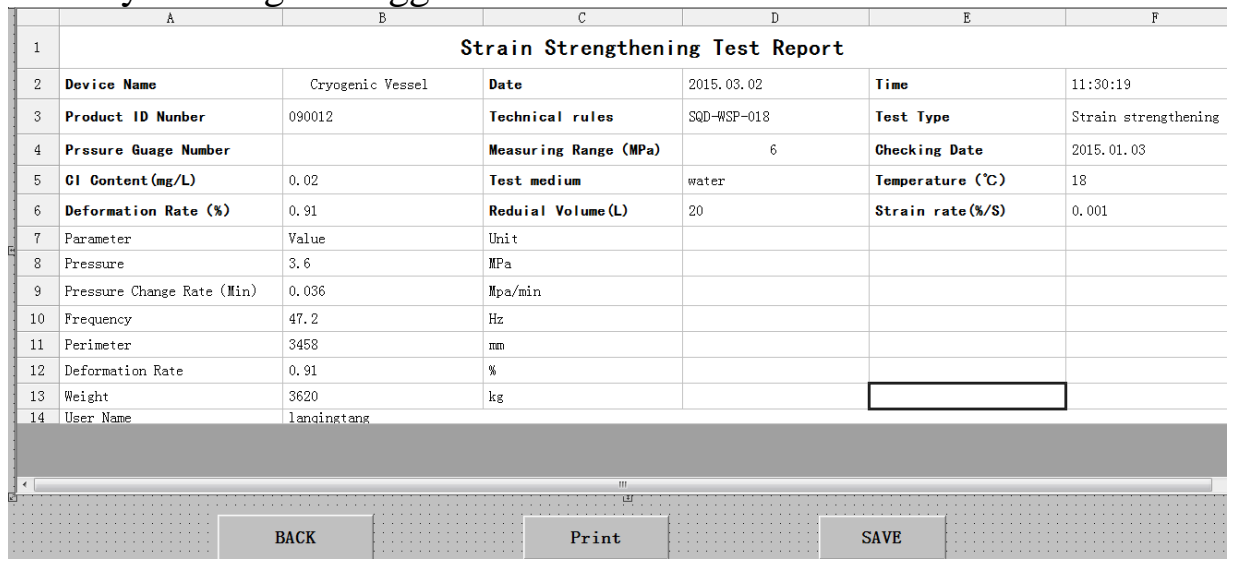

Fig.5 real-time report during strain strengthening process 


\section{PLC program design}

The actions of spot devices including valves and pumps are controlled through PLC with particular program. GX Developer is employ to design PLC program for FX2N PLC. In general, the whole program is consisted of three major parts: the main program, communication program and A/D (D/A) convert program. Fig.6 presents flow charts of the main program.

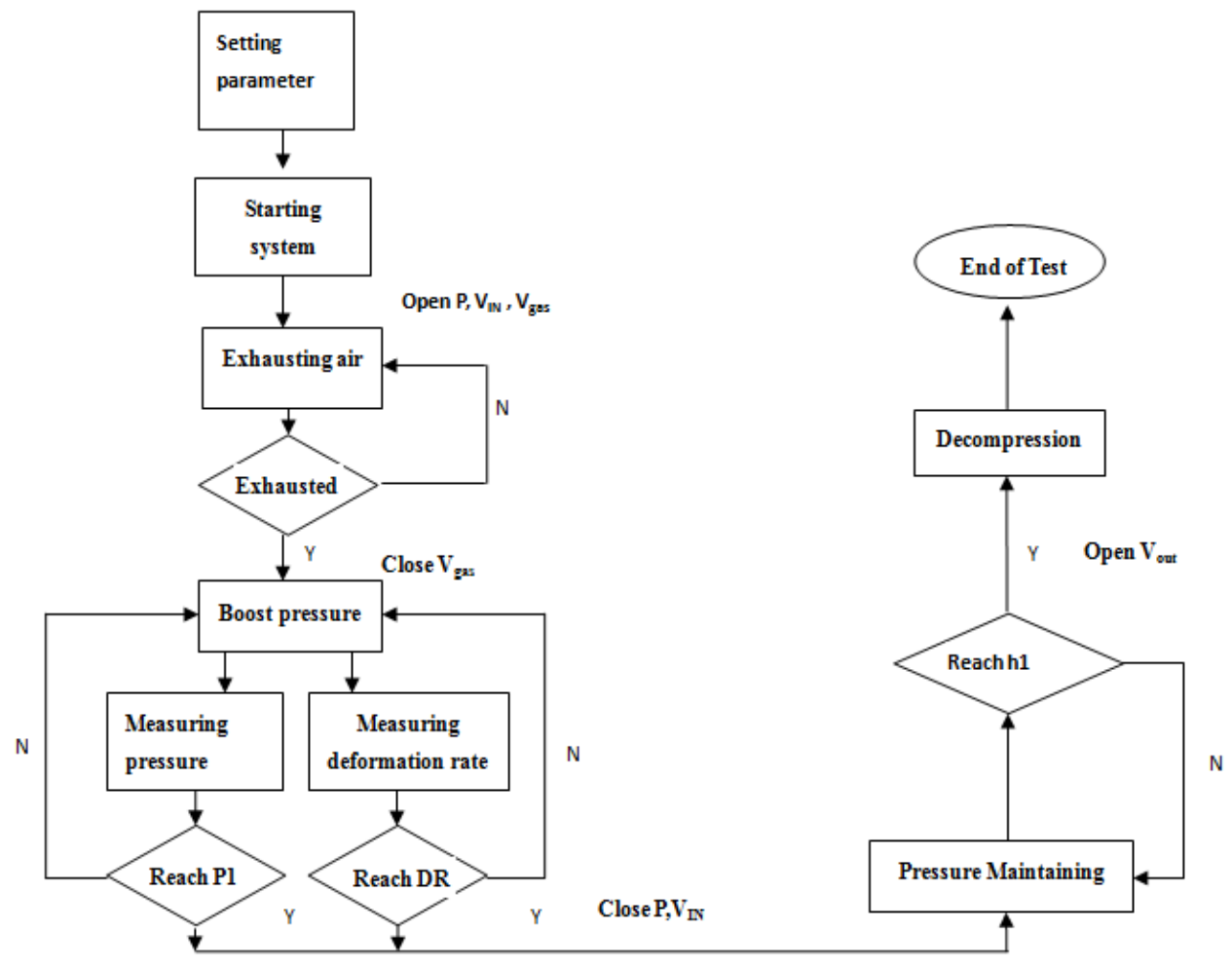

Fig. 6 flow charts of the main program.

The basic flow of strain strengthening process is as follows.

(1) Initialize and start the system, open pressure pump (P), inlet valve $\left(\mathrm{V}_{\text {in }}\right)$ and exhaust valve $\left(\mathrm{V}_{\text {gas }}\right)$ to inject water into the pressure vessel.

(2)Close exhaust valve $\left(\mathrm{V}_{\text {in }}\right)$ after discharging air completely, pressurize the vessel by pump, record pressure signal, displacement signal and weight signal gathered by sensor, convert analog signals into digital signals through $\mathrm{A} / \mathrm{D}$ converter and transform digital signals into strain-strengthening parameters such as pressure, deformation rate and residual volume.

(3)Close pressure pump $(\mathrm{P})$, inlet valve $\left(\mathrm{V}_{\text {in }}\right)$ a when pressure or deformation rate achieves the set value, maintain the system pressure with pressure-holding time.

(4)Turn on the exhaust valve $\left(\mathrm{V}_{\text {gas }}\right)$ until the holding time is over, relieve the system pressure until pressure reads zero.

(5)Save curves and reports, shut down the system finally. 
Fig.7 demonstrates a part of PLC program code.

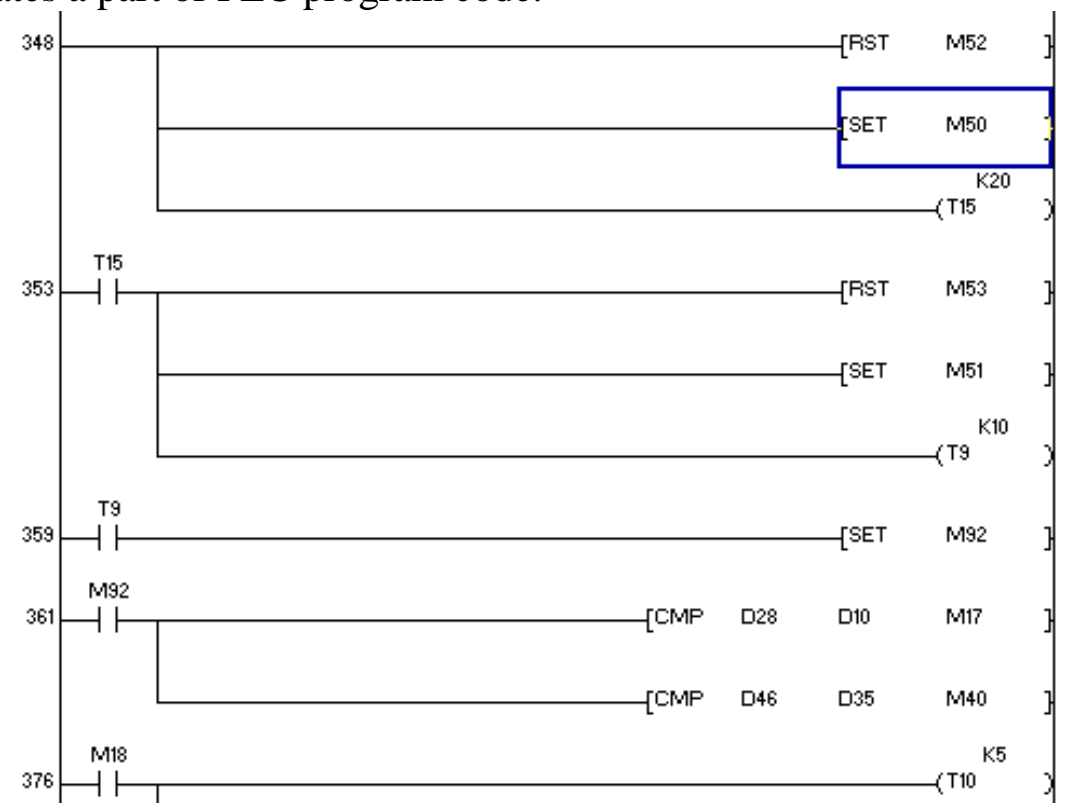

Fig.7 parts of the PLC program code

\section{System debugging}

Practical problems such as data acquisition, hardware error can be found and solved in system debug stage which can guarantee normal operation of the strain strengthening automation system.

(1) The electrical performance parameters of sensors should be calibrated for they play a significant role in system operation. The testing results reveal that the accuracy of sensor is less than $0.2 \%$ and nonlinear corrections of pressure sensor, displacement sensor and weight sensor are of good result..

(2) Several problems have been detected during the trail operations of the system. For instance, the exhaust valve did not turn on after starting the system, PLC can't receive data inputted from the parameter setting interface, history trend curves could not be displayed correctly, etc.

After multiple operations and debugging, the strain strengthening automatic control system runs well.

\section{Summary}

The operation result shows the strain strengthening automatic control system has evident stability and reliability, which can provide experience reference to the development of automatic system of the similar kind. There is no doubt that the automatic system can replace traditional strain strengthening process and liberate labor, enhance accuracy and improve efficiency.

\section{References}

[1] J.Y. Zheng, A.B Guo, C.J Miao, L. Ma, L.L Wu: Pressure Vessel. 27 (2010) 28.

[2]B.W. Wang, T. Chen, T. Xu, H. He: Eng. Fract. Mech. 37 (2013) 29.

[3]J.Y. Zheng, A.B Guo, C.J Miao, L. Ma, L.L Wu: Journal of Zhejiang University(Engineering Science). 46 (2012) 1163.

[4]H.F. Li, Z.B. Wang, X.J. Sun, C.F. Qian: Proceedings of the ASME 2013 Pressure Vessels \& Piping Division Conference. Conference, ASME, Paris (2013)43.

[5] L.Q. Tang, C.F. Qian, Z.B. Wang, H.F. Li: Eng. Fract. Mech. 134 (2015)54. 